\title{
ADDITION OF DIAZOMETHANE TO 3 AND 4-NITROPHTHALODINITRILES
}

\author{
Saida Danoun ${ }^{1}$, Genevieve Baziard-Mouysset ${ }^{1 *}$, Jean-Luc Stigliani ${ }^{~}$, Marc Payard ${ }^{1}$, \\ Mohamed Selkti ${ }^{2}$, Bernard Viossat ${ }^{2}$, Alain Tomas ${ }^{2}$, \\ ${ }^{1}$ Laboratoire de Chimie Pharmaceutique, Faculte de Pharmacie, \\ 35, chemin des Maraîchers, 31062 Toulouse Cedex 04, France. \\ 2 Laboratoire de Physique, Faculte des Sciences Pharmaceutiques et Biologiques, \\ 4, avenue de l'Observatoire, 75270 Paris Cedex 06, France.
}

\begin{abstract}
The reaction of two nitrophthalodinitriles with diazomethane is described. If the nitro group is at position 3, only one of the two nitrile functions reacts giving three isomeric $\mathrm{N}$-methyl 1,2,3-triazoles. In contrast, if the nitro group is at position 4, both nitriles react, each producing a 2-N-methyl 1,2,3-triazole. The reactivity was accounted for in terms of electronic densities, which were determined for several nitrile compounds. Interestingly, for 4-nitrophthalodinitrile, original methylation of the aromatic ring at position 5 was also observed.
\end{abstract}

\section{Introduction}

In a previous study (1) on the addition of diazomethane to different nitriles, we showed that the addition reaction requires the presence of an electroattractive group. We describe here the reactivity of the 3- and 4-nitrophthalodinitriles $\underline{2}$ and $\underline{3}$, and compare it with the unreactivity of phthalodinitrile 1 .

The nitrophthalodinitriles were stirred during several days with a solution of diazomethane in diethyl ether. The reaction was followed by thin-layer chromatography.

\section{Results}

In the case of 3-nitrophthalodinitrile 2, three $\mathrm{N}$-methyl 1,2,3-triazoles 4,5 and $\mathbf{6}$ derived from the cyano group at position 2 were isolated (cf figure 1).

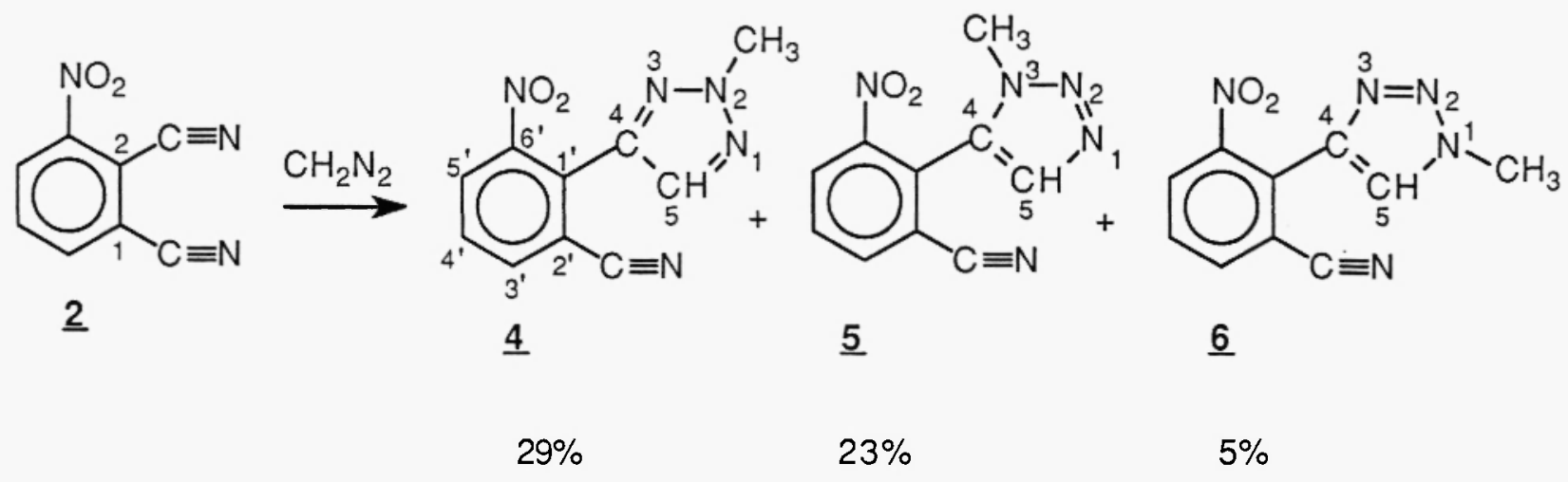

Figure 1 : (transformed product \%) 
In the case of 4-nitrophthalodinitrile 3,5 -methyl 4-nitrophthalodinitrile $\mathrm{Z}$ and two 2 -N-methyl 1,2,3triazoles $\underline{8}$ and $\underline{9}$ were isolated (cf figure 2 ).

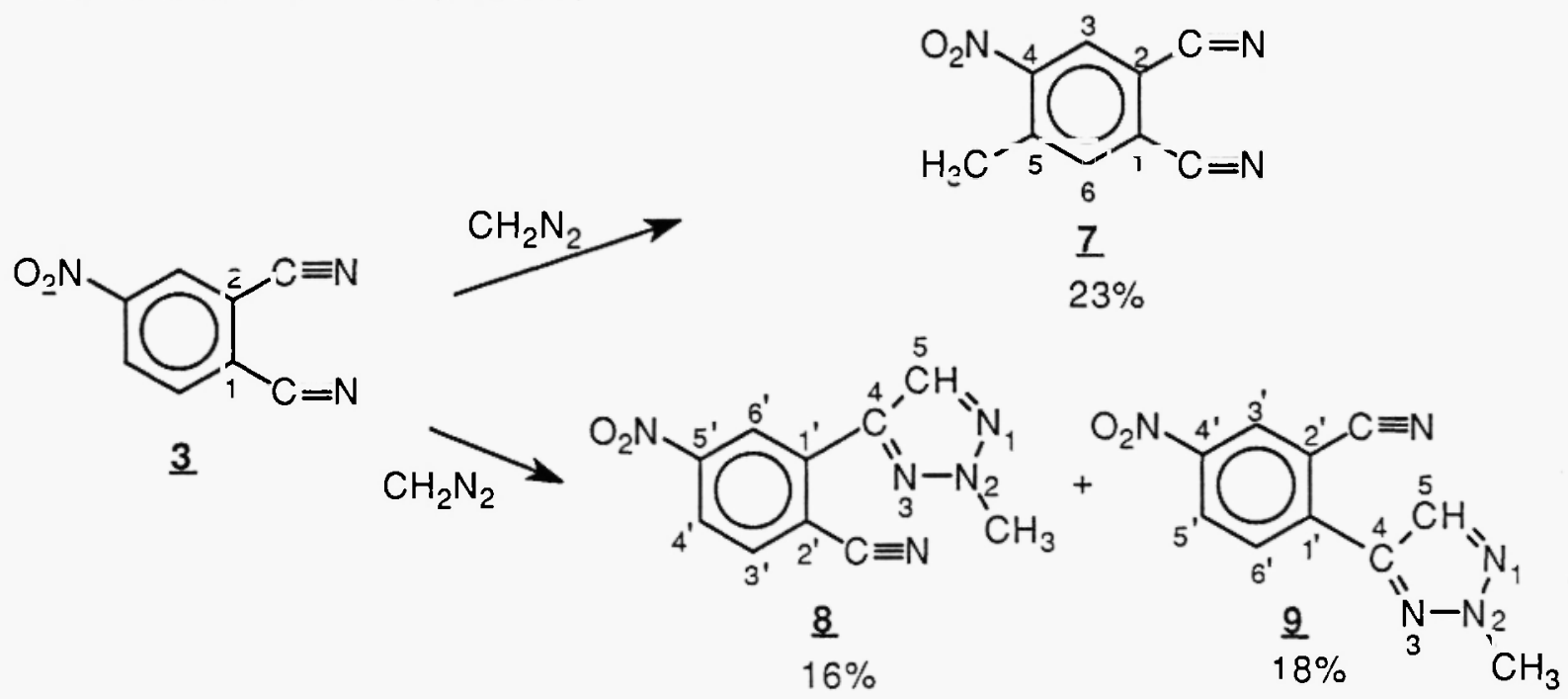

Figure 2 : (transformed product \%)

The position of the $\mathrm{N}$-methyl group on the triazole ring of compounds $\underline{4}, \underline{\mathbf{5}}, \underline{\mathbf{6}}, \mathbf{8}$ and $\underline{\mathbf{q}}$ was determined by ${ }^{13} \mathrm{C}$ NMR as described previously (1). The structures of the two isomeric $2-\mathrm{N}$-methyl-1,2,3triazoles $\underline{\varepsilon}$ and 2 were distinguished by 2D NMR (long range couplings) and confirmed by crystallographic study of compound $\mathbf{8}$ (cf figure 3 ).

5-methyl 4-nitrophthalodinitrile 7., original compound, was oblained by an unexpected methylation of the aromatic ring with diazomethane (cf figure 2). To our knowledge, the direct methylation of a benzenic ring with diazomethane has not been described, although the methylation of nitro aromatic compounds by ylides (dimethyloxosulfonium methylide or dimsyl sodium) has been reported $(2,3)$. Further the reaction of diazomethane with trinitrobenzene derivatives gives polycyclic compounds $(4,5)$, although there are numerous descriptions of $\mathrm{C}$-methylation of non-aromatic heterocyclic compounds with diazomethane (6-12).

\section{Discussion}

The electronic densities of the cyano groups can account for their reactivity towards diazomethane (13). The atomic charge on the cyano nitrogen as calculated by the AM1 method (14) must be positive for diazomethane to add to a nitrile. The net atomic charges born by the nitrogen atoms in the nitrile functions of compounds $1-9$ are listed in Table 1. 


\begin{tabular}{|c|c|c|}
\hline$\underline{\mathbf{1}}$ & $\begin{array}{c}\text { cyano group } \\
\text { position }\end{array}$ & $\begin{array}{c}\text { nitrogen net } \\
\text { atomic charge }\end{array}$ \\
\hline$\underline{\mathbf{2}}$ & $\mathbf{2}$ & $-0,009$ \\
& 1 & $-0,009$ \\
$\underline{\mathbf{3}}$ & 2 & 0,017 \\
$\mathbf{4}$ & $\mathbf{2}$ & 0,047 \\
$\mathbf{5}$ & & 0,023 \\
$\mathbf{6}$ & & 0,019 \\
& & $-0,012$ \\
$\mathbf{7}$ & & 0,006 \\
$\mathbf{8}$ & $\mathbf{2}$ & $-0,023$ \\
$\mathbf{9}$ & & 0,020 \\
\hline
\end{tabular}

Table 1 : Net atomic charge on cyano nitrogen

The phthalodinitrile 1 with a negative atomic charge $(-0.009)$ on the nitrogen atom thus does not react with diazomethane. In the same way, the reactivity of 3 and 4-nitrophthalodinitriles (compounds 2 and 3) can be related to the positive atomic charge on the nitrogen atom.

In the case of 3-nitrophthalodinitrile 2, the net atomic charge born by the nitrogen of the cyano group at position 2 is much higher than that of the cyano nitrogen at position 1, which is therefore much less reactive. Once the nitrile at position 2 has reacted, the net atomic charge on the nitrogen in the remaining nitrile (compounds 4.5 .6 ) becomes negative $(-0.012,-0.023)$ or close to zero $(0.006)$, and it does not therefore react with diazomethane.

In the case of 4-nitrophthalodinitrile 3, the two nitrile groups have nearly the same electronic densities $(0.019$ and 0.023$)$, accounting for their equivalent reactivity. As described above, once the first triazole is formed, the charge on the remaining nitrile in compounds $\underline{8}$ and $\underline{9}$ is too low for further addition to diazomethane.

The two isolated triazole compounds $\mathbf{8}$ and 9 are $\mathrm{N}$-methylated at position 2. They are generally the majority derivatives. The 1-N-methylated and $3-\mathrm{N}$-methylated isomers, which should normally be formed, could not be isolated as they were present in too low amounts. The low reactivity of compounds 2 and 3 towards diazomethane is of note. The overall converted product yields are about $55 \%$ after 15 days, and the reaction kinetics are very slow.

In other respects, the net atomic charges carried by the nitrogen atom of nitrile group of compound 7 are positive and should normally give rise to triazoles. However, the yield of the reaction with compound 7 was around $23 \%$ after 15 days. The triazoles, if formed, were present in the mixture in too small amounts to 


\section{Experimental}

The $\mathbf{3}$ - and 4-nitrophthalodinitriles $\underline{\mathbf{2}}$ and $\underline{\mathbf{3}}$ were purchased from Aldrich. Melting points were determined on a Koffler apparatus and are uncorrected. Infrared spectra were recorded on a Perkin Elmer 983-G spectrophotometer as potassium bromide pellets. The ${ }^{1} \mathrm{H}$ and ${ }^{13} \mathrm{C}$ NMR spectra were acquired on a pulsed wave spectrophotometer (Bruker $\mathrm{WH}_{250}$ ) at nominal frequencies of $250.13 \mathrm{MHz}$ for ${ }^{1} \mathrm{H}$ and 62.89 $\mathrm{MHz}$ for ${ }^{13} \mathrm{C}$. Chemical shifts were expressed in parts per million with respect to TMS. The long range couplings were determined using an AMX 400 Bruker spectrophotometer according to a previously reported method (1). Mass spectra were recorded on a Nermag R-10-10 mass spectrometer. Microanalyses for $\mathrm{C}, \mathrm{H}, \mathrm{N}$ were carried out at the Service Central d'Analyses du CNRS and results are within $0.4 \%$ of theorical values. The yields were determined by HPLC in a system including an Analprep EC 93 pump, a Shimadzu SPD2A variable wavelength ultraviolet detector and a Kromasil $\mathrm{C} 18$ reverse column.

\section{General procedure}

The 3- or 4-nitrophthalodinitrile $(5.1 \mathrm{~g}, 0.03 \mathrm{~mole})$ was added to a solution of diazomethane $(5.5 \mathrm{~g}$, $0.13 \mathrm{~mole}$ ) in diethyl ether $(600 \mathrm{ml})$, prepared according to Boer's method (15). The mixture was maintained at room temperature for a week. Further diazomethane $(5.5 \mathrm{~g}, 0.13$ mole) was then added as not all nitriles had reacted. The mixture was maintained for a further week at room temperature. Although nitriles had not completely reacted, the solvent was evaporated and the residue was chromatographed on a silica gel column (70-230 mesh).

\section{Compounds 4,5 and 6 :}

Two successive columns with cyclohexane / ethylacetate $(70 / 30)$ as eluent effected the separation of the three compounds $4, \underline{5}$ and $\underline{6}$.

\section{- 4-(2-cyano 6-nitrophenyl) 2-N-methyl v-triazole 4 :}

$M p=107^{\circ} \mathrm{C} . R f=0.26$ (dichloromethane). $M S, m / z: 229.1\left(M^{+}\right.$).

IR $\left(\mathrm{KBr}, \mathrm{cm}^{-1}\right): 3083,3043,2879(\mathrm{C}-\mathrm{H}) ; 2239(\mathrm{CN}) ; 1542,1353\left(\mathrm{NO}_{2}\right)$.

${ }^{1} \mathrm{H} \mathrm{NMR}\left(\mathrm{CDCl}_{3}, \delta \mathrm{ppm}\right): 4.26\left(\mathrm{~s}, 3 \mathrm{H}, \mathrm{CH}_{3}\right) ; 7.67(\mathrm{~m}, 1 \mathrm{H}, \mathrm{H}-4) ; 7.88(\mathrm{dd}, 1 \mathrm{H}, \mathrm{H}-3$ ',$J=7.9$ and $1.4 \mathrm{~Hz}) ; 7.92$ $(\mathrm{s}, 1 \mathrm{H}, \mathrm{H}-5) ; 8.03(\mathrm{dd}, \mathrm{tH}, \mathrm{H}-5, \mathrm{~J}=8.2$ and $1.3 \mathrm{~Hz}$ ).

${ }^{13} \mathrm{C} \mathrm{NMR}\left(\mathrm{CDCl}_{3}, \delta \mathrm{ppm}\right): 42.17\left(\mathrm{CH}_{3}\right) ; 115.22(\mathrm{C}-2) ; 116.01(\mathrm{CN}) ; 127.95$ (dd, C-5', J C-H = $171.2 \mathrm{~Hz}$ and $7.5 \mathrm{~Hz}$ ); 128.28 (C-1'); 130.04 (d, C-4', J C-H = 169.5 Hz); 134.17 (d, C-5, J C-H = 196 Hz); 136.7 (dd, C-3' , $J \mathrm{C}-\mathrm{H}=169.7 \mathrm{~Hz}$ and $7.7 \mathrm{~Hz}) ; 138.96(\mathrm{C}-4) ; 149.98\left(\mathrm{C}-6^{\prime}\right)$.

\section{- 4-(2-cyano 6-nitrophenyl) 3-N-methyl v-triazole 5 :}

$\mathrm{Mp}=166^{\circ} \mathrm{C} . \mathrm{Rf}=0.21$ (dichloromethane). MS, $m / z: 229.1\left(\mathrm{M}^{+}\right)$.

IR $\left(\mathrm{KBr}, \mathrm{cm}^{-1}\right): 3133,3071,2960(\mathrm{C}-\mathrm{H}) ; 2237(\mathrm{CN}) ; 1585,1355\left(\mathrm{NO}_{2}\right)$.

${ }^{1} \mathrm{H} \mathrm{NMR}\left(\mathrm{CDCl}_{3}, \delta \mathrm{ppm}\right): 4.30\left(\mathrm{~s}, 3 \mathrm{H}, \mathrm{CH}_{3}\right) ; 7.82(\mathrm{~m}, 1 \mathrm{H}, \mathrm{H}-4) ; 8.23\left(\mathrm{dd}, 1 \mathrm{H}, \mathrm{H}-3^{\prime}, J=8.2\right.$ and $\left.1.2 \mathrm{~Hz}\right) ; 8.32$ (dd, $1 H, H-5, J=8.1$ and $1.3 \mathrm{~Hz}$ ); $8.39(\mathrm{~s}, 1 \mathrm{H}, \mathrm{H}-5$ ). 
${ }^{13} \mathrm{C} \mathrm{NMR}\left(\mathrm{CDCl}_{3}, \delta \mathrm{ppm}\right): 42.19\left(\mathrm{CH}_{3}\right) ; 114.18(\mathrm{CN}) ; 118.67\left(\mathrm{C}-2^{\prime}\right) ; 124.68$ (dd, C-5', J C-H = $172.2 \mathrm{~Hz}$ and $8.2 \mathrm{~Hz}$ ); 126.05 (C-1'); 131.85 (C-4); 133.27 (d, C-4', J C-H = $166.8 \mathrm{~Hz}$ ); 133.92 (dd, C-3', J C-H = $161.8 \mathrm{~Hz}$ and $7.5 \mathrm{~Hz}) ; 133.92(\mathrm{~d}, \mathrm{C}-5, J \mathrm{C}-\mathrm{H}=195.6 \mathrm{~Hz}) ; 142.50\left(\mathrm{C}-6^{\prime}\right)$.

- 4-(2-cyano 6-nitrophenyl) 1-N-methyl v-triazole $\underline{6}$ :

$\mathrm{Mp}=179^{\circ} \mathrm{C} . \mathrm{Rf}=0.11$ (dichloromethane). $\mathrm{MS}, \mathrm{m} / z: 229.1\left(\mathrm{M}^{+}\right)$.

IR $\left(\mathrm{KBr}, \mathrm{cm}^{-1}\right): 3155,3097,2925(\mathrm{C}-\mathrm{H}) ; 2233(\mathrm{CN}) ; 1527,1347\left(\mathrm{NO}_{2}\right)$.

$1^{1} \mathrm{H} \mathrm{NMR}\left(\mathrm{CDCl}_{3}, \delta \mathrm{ppm}\right): 4.22\left(\mathrm{~s}, 3 \mathrm{H}, \mathrm{CH}_{3}\right) ; 7.87(\mathrm{~m}, 1 \mathrm{H}, \mathrm{H}-4$ ) $) ; 8.24\left(\mathrm{dd}, 1 \mathrm{H}, \mathrm{H}-3^{\prime}, J=8.2\right.$ and $1.2 \mathrm{~Hz}$ ); 8.59 (s, $1 \mathrm{H}, \mathrm{H}-5) ; 8.78$ (dd, $1 \mathrm{H}, \mathrm{H}-5, \mathrm{~J}=8.1$ and $1.2 \mathrm{~Hz}$ ).

${ }^{13} \mathrm{C} \mathrm{NMR}\left(\mathrm{CDCl}_{3}, \delta \mathrm{ppm}\right): 37.00\left(\mathrm{CH}_{3}\right) ; 114.84(\mathrm{CN}) ; 124.29$ (dd, $\mathrm{C}-5$ ', $J \mathrm{C}-\mathrm{H}=174 \mathrm{~Hz}$ and $8.4 \mathrm{~Hz}$ ); 124.60 (d, C-5, J C-H = 196.3 Hz); $126.60\left(\mathrm{C}^{\prime} 2^{\prime}\right) ; 127.01\left(\mathrm{C}-1^{\prime}\right) ; 133.30\left(\mathrm{~d}, \mathrm{C}-4^{\prime}, J \mathrm{C}-\mathrm{H}=169.3 \mathrm{~Hz}\right.$ ); 133.38 (dd, C$3^{\prime}, J \mathrm{C}-\mathrm{H}=168.8 \mathrm{~Hz}$ and $\left.7.5 \mathrm{~Hz}\right) ; 137.28(\mathrm{C}-4) ; 141.87\left(\mathrm{C}^{-} 6^{\prime}\right)$.

Compounds $\underline{7}, \underline{8}$ and $\underline{9}$ :

A first column with a mixture of dichloromethane / cyclohexane $(50 / 50)$ as eluent gave the compound $\underline{7}$ and a mixture of compounds $\underline{8}$ and 9 . A second column with cyclohexane / ethyl acetate $(80 / 20)$ as eluent effected the separation of the two other compounds $\underline{8}$ and $\underline{9}$.

\section{- 5-Methyl-4-nitrophthalodinitrile 7 :}

$\mathrm{Mp}=110^{\circ} \mathrm{C} . \mathrm{Rf}=0.55$ (dichloromethane). $\mathrm{MS}, m / z: 187.0\left(\mathrm{M}^{+}\right)$.

IR $\left(\mathrm{KBr}, \mathrm{cm}^{-1}\right): 3081,2922(\mathrm{C}-\mathrm{H}) ; 2240(\mathrm{CN}) ; 1521,1350\left(\mathrm{NO}_{2}\right)$;

${ }_{1}^{1} \mathrm{H} \mathrm{NMR}\left(\mathrm{CDCl}_{3}, 250.13 \mathrm{MHz}\right): 2.72\left(\mathrm{~s}, 3 \mathrm{H}, \mathrm{CH}_{3}\right) ; 7.84(\mathrm{~s}, 1 \mathrm{H}, \mathrm{H}-6) ; 8.34(\mathrm{~s}, 1 \mathrm{H}, \mathrm{H}-3)$.

${ }_{13} \mathrm{C} \mathrm{NMR}\left(\mathrm{CDCl}_{3}, 63.89 \mathrm{MHz}\right): 20.34\left(q, \mathrm{CH}_{3}, J \mathrm{C}-\mathrm{H}=131.6 \mathrm{~Hz}\right) ; 113.44$ and $113.66(\mathrm{CN}) ; 114.61(\mathrm{C}-2) ;$ $119.10(C-1) ; 129.25$ (d, C-3, J C-H = 174.6 Hz); 138.08 (d, C-6, J C-H = 170.9 Hz); 139.68 (C-5); 150.74 (C-4).

- 4-(2'-cyano-5'-nitrophenyl)-2-N-methyl-1,2,3-triazole 8 :

$\mathrm{Mp}=149^{\circ} \mathrm{C} . \mathrm{Rf}=0.30$ (dichloromethane). MS, $m / z: 229.1\left(\mathrm{M}^{+}\right)$.

IR $\left(\mathrm{KBr}, \mathrm{Cm}^{-1}\right)$ : 3133, 3067, $2961(\mathrm{C}-\mathrm{H}) ; 2232(\mathrm{CN}) ; 1519,1353\left(\mathrm{NO}_{2}\right)$;

${ }^{1} \mathrm{H} \mathrm{NMR}\left(\mathrm{CDCl}_{3}, 250.13 \mathrm{MHz}\right): 4.31\left(\mathrm{~s}, 3 \mathrm{H}, \mathrm{CH}_{3}\right) ; 7.94\left(\mathrm{~d}, 1 \mathrm{H}, \mathrm{H}-3^{\prime}, J=8.5 \mathrm{~Hz}\right) ; 8.25\left(\mathrm{dd}, 1 \mathrm{H}, \mathrm{H}-4^{\prime}, J=8.5\right.$ and $2.2 \mathrm{~Hz}$ ); $8.34(\mathrm{~s}, 1 \mathrm{H}, \mathrm{H}-5) ; 8.81\left(\mathrm{~d}, 1 \mathrm{H}, \mathrm{H}-6^{\circ}, J=2.2 \mathrm{~Hz}\right.$ ).

${ }^{13} \mathrm{C} \mathrm{NMR}\left(\mathrm{CDCl}_{3}, 63.89 \mathrm{MHz}\right): 42.12$ (q, $\mathrm{CH}_{3} . J \mathrm{C}-\mathrm{H}=142.5 \mathrm{~Hz}$ ); $114.24(\mathrm{C}-1$ ) $) ; 16.82(\mathrm{CN}) ; 122.40$ (dd, C$4^{\prime}, J \mathrm{C}, \mathrm{H}=175.2$ and $5.27 \mathrm{~Hz}$ ); 123.04 (dd, C-6', J C-H = 172.9 and $3.9 \mathrm{~Hz}$ ); $133.03(\mathrm{~d}, \mathrm{C}-5, J \mathrm{C}-\mathrm{H}=195.1$ $\mathrm{Hz}) ; 135.15\left(\mathrm{~d}, \mathrm{C}-3^{\prime}, J \mathrm{C}-\mathrm{H}=171.8 \mathrm{~Hz}\right) ; 135.48$ (C-2'); $142.23(\mathrm{C}-4) ; 150.09$ (C-5').

- 4-(2'-cyano-4'-nitrophenyl)-2-N-methyl-1,2,3-triazole 9 :

$\mathrm{Mp}=131^{\circ} \mathrm{C} . \mathrm{Rf}=0.28$ (dichloromethane). MS, $m / z: 229.1\left(\mathrm{M}^{+}\right)$.

IR $\left(\mathrm{KBr}, \mathrm{Cm}^{-1}\right) \cdot 3155,3069,2959(\mathrm{C}-\mathrm{H}) ; 2235(\mathrm{CN}) ; 1528,1357\left(\mathrm{NO}_{2}\right)$

${ }^{1} \mathrm{H} \mathrm{NMR}\left(\mathrm{CDCl}_{3}, 250.13 \mathrm{MHz}\right): 4.41\left(\mathrm{~s}, 3 \mathrm{H}, \mathrm{CH}_{3}\right) ; 8.23\left(\mathrm{~d}, 1 \mathrm{H}, \mathrm{H}-6{ }^{\prime}, J=8.8 \mathrm{~Hz}\right) ; 8.39(\mathrm{~s}, 1 \mathrm{H}$, 
${ }^{13} \mathrm{C} \mathrm{NMR}\left(\mathrm{CDCl}_{3}, 63.89 \mathrm{MHz}\right): 42.29\left(\mathrm{q}, \mathrm{CH}_{3} . \mathrm{J} \mathrm{C}-\mathrm{H}=142.45 \mathrm{~Hz}\right) ; 110.12(\mathrm{C}-1) ; 116.72(\mathrm{CN}) ; 127.60$ (d, $C^{-5}, J \mathrm{C}-\mathrm{H}=168.13 \mathrm{~Hz}$ ); 129.19 (d, C-6', J C-H = 173.2 Hz); 129.21 (d, C-3', J = 167.5 Hz); 133.78 (d, C-5, $J \mathrm{C}-\mathrm{H}=195.4 \mathrm{~Hz}) ; 139.04\left(\mathrm{C}-2^{\prime}\right) ; 142.37(\mathrm{C}-4) ; 146.69\left(\mathrm{C}-4^{\prime}\right)$.

\section{X-ray analysis}

Crystal data of the compound $8: \mathrm{C}_{10} \mathrm{H}_{7} \mathrm{~N}_{5} \mathrm{O}_{2} \cdot \mathrm{M}_{W}=229.20$. A suitable crystal was investigated on a Siemens P3 diffractometer (Mo K $\alpha$ radiation $=0.71073 \AA$, graphite monochromator). Triclinic, space group $\mathrm{P} \overline{1}, \mathrm{Z}=2, \mathrm{a}=7.217(2) \AA, \mathrm{b}=7.989(6) \AA, c=9.898(8) \AA, \alpha=76.63(6)^{\circ}, \beta=85.15(5)^{\circ}, \gamma=67.79(4)^{\circ}, V=$ $514.0(6) \AA^{3}, d_{x}=1.47 \mathrm{~g} . \mathrm{cm}^{-3}, F(000)=236, \mu=0.11 \mathrm{~mm}^{-1} .2371$ unique reflections measured up to $2 \theta=$ $55.10^{\circ}$ of which 1278 with $\mathrm{I}>3 \sigma(\mathrm{l})$ were kept in refinement calculations. The structure was solved by direct methods using SHELXS86 (16) and refined by full matrix least-squares with SHELX76 (17) minimizing the quantity $\Sigma w\left(F_{O}-F_{C}\right)^{2}$. Non hydrogen atoms were refined with anisotropic temperature factors; hydrogen atoms were located in difference Fourier map at observed positions. Convergence was reached at $R=$ 0.059 . The residual electron density in the final difference Fourier map shows no features up to 0.26 e. $\AA^{-3}$. Lists of the fractional atomic coordinates are given in table 2.

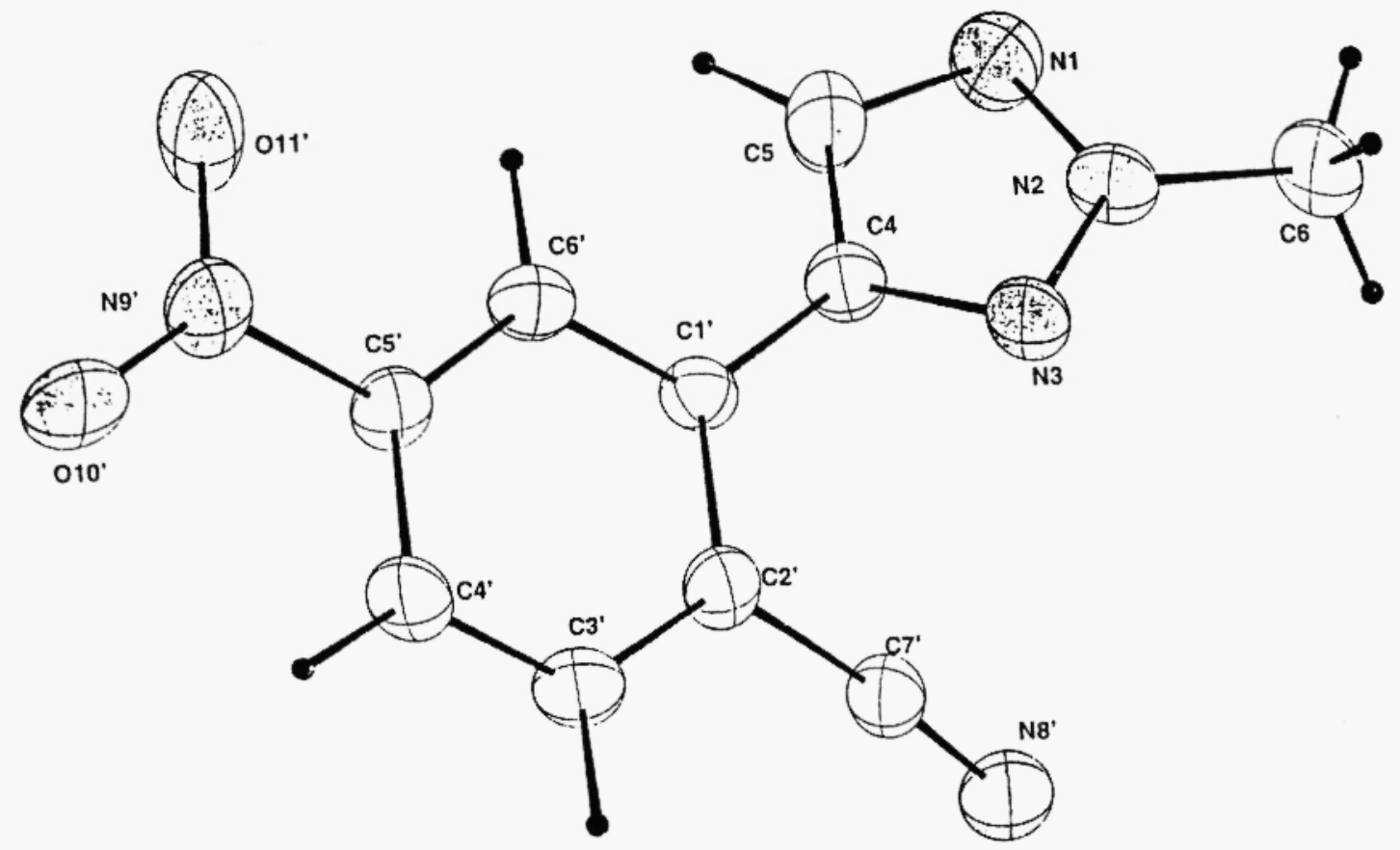

Figure 3 : ORTEP view of 8 
Table 2 : Positionnal parameters and mean recalculated isotropic for non-hydrogen atoms

\begin{tabular}{|c|c|c|c|c|c|c|c|c|}
\hline TOM & $\mathrm{x}$ & & $Y$ & & $\mathrm{z}$ & & $\mathrm{U}_{\text {eq }}$ & \\
\hline$C\left(1^{\prime}\right)$ & 589 & ( 5) & -0.0043 & ( 5) & 0.5920 & $(4)$ & 0.0370 & \\
\hline & 728 & ( 5) & -0.1460 & 15 & .5250 & $(4)$ & 0370 & r \\
\hline Cl & .2525 & $(6)$ & -0.1105 & $(6)$ & 3811 & (4) & 0490 & 1 \\
\hline Cl & 133 & ( 6) & 0.0644 & 16 & 017 & (4) & 460 & i \\
\hline & 997 & (6) & 026 & 15 & 88 & $(4)$ & 390 & i \\
\hline C & 18 & $(6)$ & 0.1721 & ( 5) & 098 & (4) & 0.0430 & $i$ \\
\hline Cl & .3011 & ( 6) & -0.3327 & $(6)$ & 0.5998 & $(4)$ & 0.0450 & i \\
\hline Ni & .3160 & (6) & -0.4812 & (5) & .6508 & (4) & 0.0620 & i \\
\hline & .1591 & ( 5) & 3919 & 15 & 58 & $(4)$ & 0.0490 & i \\
\hline & .1159 & ( 5$)$ & .4237 & 15 & 41 & (3) & 80 & i \\
\hline 01 & 680 & ( 5) & 0.5075 & (4) & 35 & $(4)$ & 0.0740 & i \\
\hline N ( & 0.2557 & ( 6) & 0.0032 & ( 5) & 86 & (4) & 0.0620 & 1 \\
\hline No & 0.3789 & ( 5) & -0.1644 & ( 5) & 0.9458 & $(3)$ & 0.0510 & \\
\hline Ni & 0.3977 & ( 5) & -0.1950 & i 5 & .8188 & (3) & 0.0460 & \\
\hline & .2786 & ( 6) & -0.0340 & $(5$ & 24 & $(4)$ & 0.0400 & \\
\hline & & ( 7) & & $(7$ & & & 0.0580 & \\
\hline$C(6)$ & 0.4848 & (9) & -0.3037 & ( 7 ) & 1.0645 & ( 5) & 0.0650 & \\
\hline
\end{tabular}

Complete data have been deposited with the editorial office.

\section{References}

(1) S.Danoun, G. Baziard-Mouysset, J.L.Stigliani, G. Commenges, A. Carpy, M. Payard, Bull. Soc. Chim. Fr. 132, 943 (1995)

(2) V.J. Traynalis, J.V. Mc Sweeney, J. Org. Chem. 31, 243 (1966)

(3) G.A. Russel , S.A. Weiner , J. Org. Chem. 31, 248 (1966)

(4) H. V. Pechmann, Ber. Dtsch. Chem. Ges. 33, 627 (1900)

(5) J.C. Van Velzen, C. Kruk, T.J. De Boer, Rec. Trav. Chim. Pays-Bas 90, 842 (1971)

(6) M. Alguero, J. Bosch , J. Castaner, J. Castella, J. Castells, R. Mestres, J. Pascual, F. Serratosa , Tetrahedron 18, 1381 (1962)

(7) J. Hamelin , R. Carrie, Bull. Soc. Chim. Fr. 5, 2162 (1968)

(8) R. Clinging , F.M. Dean, L.E. Houghton, J. Chem. Soc. (C) 897(1970)

(9) J.S. Pizey, Synthetic Reagents, vol II, Wiley, Ed. Ellis Horwood Limited, New-York, (1974)

(10) J.H. Lister, Aust. J. Chem. 32, 2771 (1979)

(11) F.M. Dean, R.S. Johnson, J. Chem. Soc. Perkin Trans. I 224 (1981)

(12) H. Laatsch, Liebigs Ann. Chem. 299 (1983)

(13) S. Danoun, Thèse Doctorat es Sciences Pharmaceutiques,

Universite Paul Sabatier (Toulouse), n²502(1996)

(14) M.J.S. Dewar, E.G. Zoebisch, E.F. Healy, J.J.P. Stewart, J. Am. Chem. Soc.107, 3902 (1985)

(15) T.J. Boer, H.J. Baker, Organic Synth. 4, 250 (1963)

(16) G.M. Sheldrick, SHELXS86. Program for the solution of crystal structures.

Univ. of Göttingen, Germany, (1986).

(17) G.M. Sheldrick, SHELX76. Program for crystal structure determination.

Univ. of Cambridge, England, (1976).

Received on September 7, 1997 
